\title{
Michelson-interferometer-based method for measuring the angle of rotation
}

Cexiang Foo, Gaurav Madan, Theresa Lai, Mark Wee, Yu Fu, et al.

Cexiang Foo, Gaurav Madan, Theresa Lai, Mark Wee, Yu Fu, Huai Min Shang, "Michelson-interferometer-based method for measuring the angle of rotation," Proc. SPIE 4588, Seventh International Conference on Education and Training in Optics and Photonics, (28 May 2002); doi: 10.1117/12.468719

Event: Education and Training in Optics and Photonics 2001, 2001, Singapore, Singapore 


\title{
A Michelson Interferometer-Based Method For Measuring The Angle Of Rotation
}

\author{
Foo Cexiang ${ }^{\mathrm{a}}$, Gaurav Madan ${ }^{\mathrm{a}}$, Theresa Lai ${ }^{\mathrm{a}}$, Mark Wee ${ }^{\mathrm{a}}$, Y. Fu ${ }^{\mathrm{b}}$, H.M. Shang ${ }^{\mathrm{b} *}$ \\ ${ }^{a}$ Raffles Institution, 1 Raffles Institution Lane, Singapore 575954 \\ ${ }^{b}$ Department of Mechanical Engineering, National University of Singapore, 10 Kent Ridge Crescent, \\ Singapore 119260 \\ *Email: mpeshm@nus.edu.sg
}

\begin{abstract}
For measuring the angle of rotation of flat objects using projected fringes, the method of point-of-light triangulation and the method of line-of-light triangulation will breakdown when the grating lies on the axis of rotation. Therefore, a grating other than a point or linear lines is preferred. In this paper, a simple Michelson interferometer-based method for the generation and projection of circular gratings is described. The basic optical element in a Michelson interferometer is a beam-splitting cube. With this Michelson interferometer, a circular grating is observed when the screen is placed normal to the line containing the two point-light sources produced by the beam splitter. By placing an expander between the beam-splitter and the laser source, and by carefully adjusting the two mirrors beside the beam-splitting cube, the frequency of the circular grating can be adjusted. This paper also describes the use of the generated circular optical grating for measuring the amount of rotation of flat surfaced that are either diffuse or specularly reflective - the method is based on relating the distortion of the circular grating to the angular rotation of the surface.
\end{abstract}

Keywords: circular grating, Michelson interferometer, fringe projection, measurement of angle of rotation

\section{INTRODUCTION}

The angle of rotation of a surface about its axis can be measured in a number of ways based on optical interferometry, internal-reflection of optical elements and fringe projection. ${ }^{1-10}$ Each method has its advantages and limitations. For example, the method of point-of-light triangulation is easy to perform, requiring only a simple optical arrangement; but if the illuminating point lies on the axis of rotation, the rotating angle of a diffuse surface cannot be determined unless three non-collinear illuminating points are used. In the fringe projection method, the change in pitch of the linear fringes on the rotating surface is related to the rotating angle. However, the angle of rotation cannot be determined if the direction of the fringes happens to be perpendicular to the axis of rotation.

This paper describes an alternative method for measuring small angles of rotation of a flat surface using a circular optical grating. The main advantage of this method lies in its ability to determine the angle of rotation when the flat surface rotates about any axis. Furthermore, the method can be used on specimens with diffused surfaces and specimens with specularly reflective surfaces. For object with a diffused surface, the distorted grating is recorded off the object surface. For objects with a specularly reflective surface, the distorted grating is specularly reflected onto an opaque screen and the $\mathrm{CCD}$ camera subsequently records the grating image off this screen.

\section{GENERATION OF CIRCULAR GRATING}

Figure 1 illustrates the optical arrangement of a Mechelson interferometer for the generation and projection of optical circular grating. By carefully adjusting the mirrors so that the two light beams exiting from the beam-splitting cube are collinear (but with optical path difference) and normal to a flat screen, a circular grating will be generated and projected on the screen. Suppose the two light wavefronts exiting from the beam-splitting cube are emanated from point-light sources $\mathrm{S}(0,0, D)$ and $\mathrm{S}^{\prime}\left(0,0, D+d_{z}\right)$ located on the reference z-axis. It can easily be shown that the typical point $P_{0}\left(x_{0}, y\right.$, 0 ) on a locus of constant phase-difference is described by the following expression. 
$x^{2}+y^{2}=\frac{\left(d_{z}^{2}-\Delta^{2}\right)^{2}+4 D\left(d_{z}^{2}-\Delta^{2}\right)\left(D+d_{z}\right)}{4 \Delta^{2}}$

where $\Delta$ is related to the fringe order $N$ via the well-known relation $\Delta=\frac{\lambda}{2} N$, with $\lambda$ denoting the wavelength of the laser source and $N$ denoting the fringe order. By placing a converging lens between the beam-splitting cube and the test surface, the diameter of the optical grating may either be magnified or be reduced.

Screen or flat surface

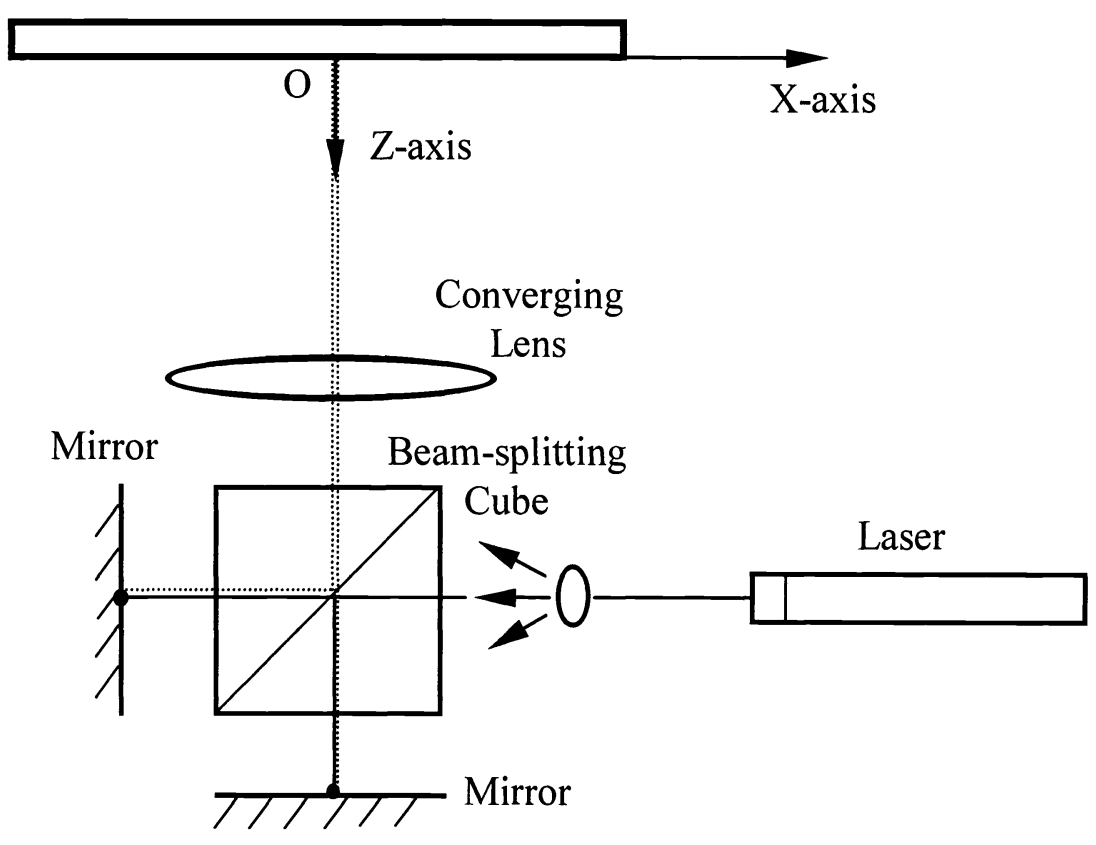

Fig. 1. Generation of optical grating using Michelson Interferometer

\section{MEASUREMENT OF THE ROTATING ANGLE OF A DIFFUSE SURFACE}

Suppose the same interference pattern generated by point-light sources $S(0,0, D)$ and $S^{\prime}\left(0,0, D+d_{z}\right)$ and expressed by Eq. (1) is now projected onto a planar mirror surface that is inclined at the angle $\theta=\theta_{1}$ (Figure 2). It is readily derived that the interference pattern appearing on the mirror surface is described by the following expression.

$$
\begin{aligned}
x^{2}+y^{2}=\frac{\left(d_{z}^{2}-\Delta^{2}\right)^{2}+4 D\left(d_{z}^{2}-\Delta^{2}\right)\left(D+d_{z}\right)}{4 \Delta^{2}}+ \\
\quad\left[\frac{d_{z}^{2}-\Delta^{2}}{\Delta^{2}}\right] Z^{2}-\left[\frac{\left(d_{z}^{2}-\Delta^{2}\right)\left(2 D+d_{z}\right)}{\Delta^{2}}\right] Z
\end{aligned}
$$

where $Z$, the z-coordinate of any point $P(x, y, Z)$ on an interference fringe that is projected on the inclined surface, is given as $Z=x \tan \theta$. 


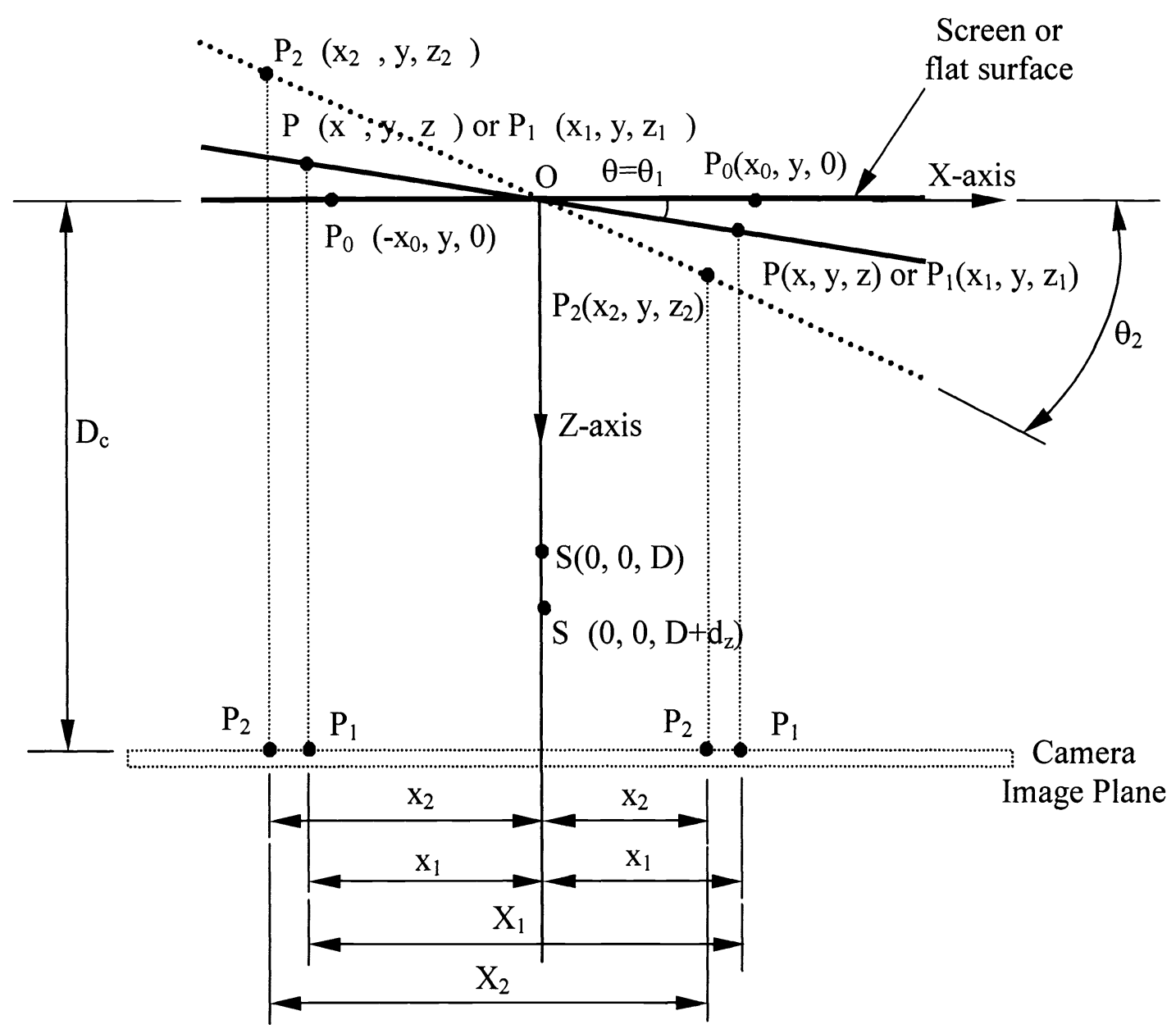

Fig. 2. Determination of the rotating angle from the distorted circular grating that appears on a diffuse/specularly reflective surface.

Suppose the image of the circular grating that appears on the mirror surface is recorded with a photographic film or a CCD camera whose direction of recording is parallel with the reference $z$-axis. The length of a fringe-circle on the $x y$ plane $(\theta=0)$ is denoted by $X=2 x_{0}$ in a direction parallel with the $x$-axis, and by $Y$ in a direction parallel with the $y$-axis. Suppose the test surface is slightly rotated about the $y$-axis from the initial angle $\theta_{l}$ to angle $\theta_{2}$, then the value of $X$ is changed from $X_{1}$ to $X_{2}$ whereas the value of $\mathrm{Y}$ is unchanged throughout the angular change. For two distantly located but closely spaced point-light sources $\mathrm{S}(0,0, D)$ and $\mathrm{S}^{\prime}\left(0,0, D+d_{z}\right)$, and for small angle of rotation of a relatively small object surface, it can be shown that the rotating angle may be determined from the distortion of the circular grating using the following expression.

$\theta_{2}-\theta_{1} \approx-\frac{K}{2}\left(\frac{X_{2}-X_{1}}{Y}\right)=-\frac{K}{2} \psi$

where $K \equiv \frac{Y}{D \xi}$ is defined as a sensitivity factor related to the optical arrangement; and $\xi$, which is related to the phasedifference $\Delta$ (or fringe order $\mathrm{N}$ ), is defined as $\xi \equiv\left(\frac{d_{c}{ }^{2}-\Delta^{2}}{\Delta^{2}}\right)$. 


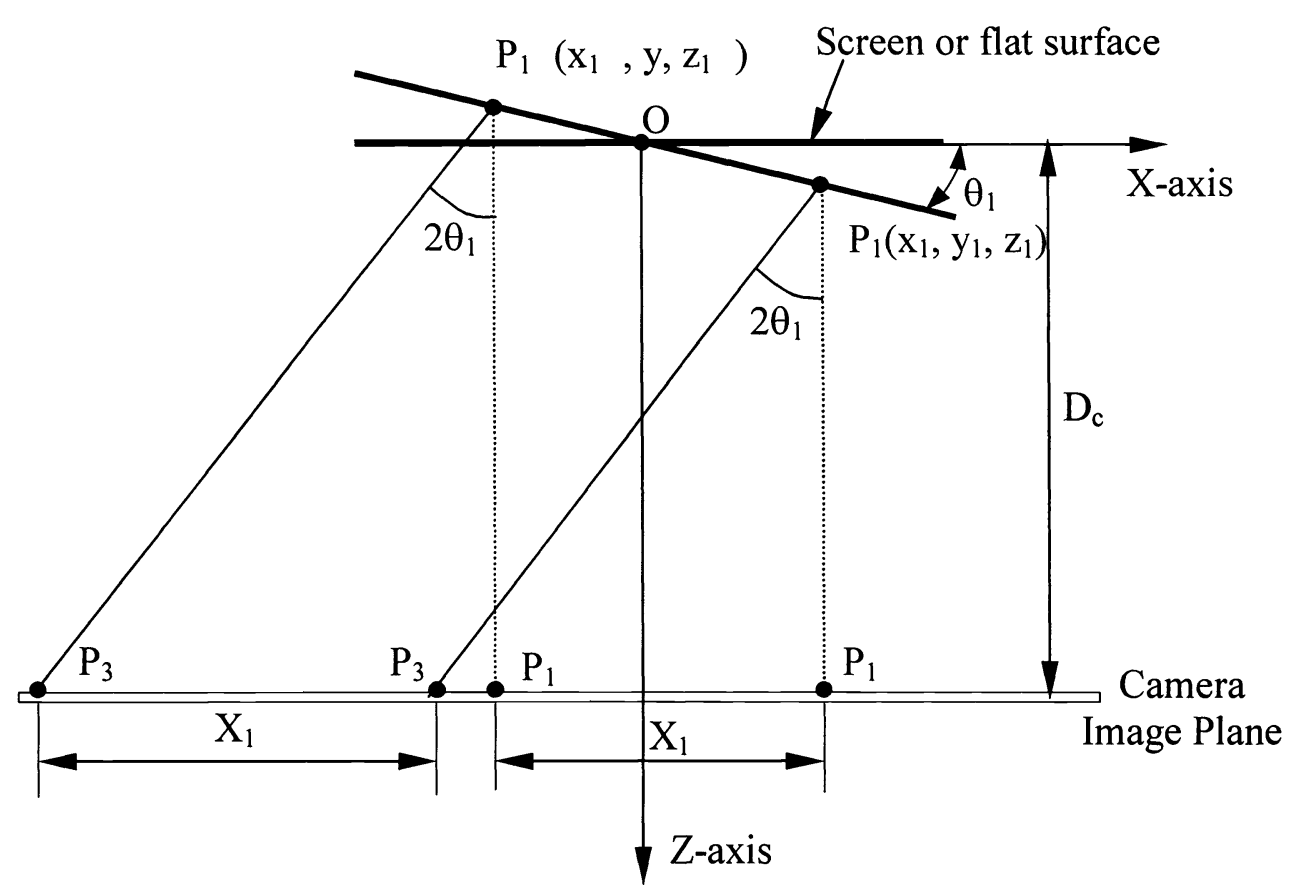

Fig. 3. Determination of the rotating angle from the distorted grating that is reflected from a mirror-like surface

\section{MEASUREMENT OF THE ROTATING ANGLE OF A MIRROR-LIKE SURFACE}

The rotating angle of a specularly reflective surface can be measured in the following manner: the image of the grating that is projected onto the surface is reflected onto an opaque screen, after which the distorted image is recorded off this screen. Figure 3 shows the schematic layout of this method. The optical grating is projected along $\mathrm{z}$-axis onto a mirror surface that is inclined at the angle $\theta_{1}$ with x-axis. A fringe-circle that is cast on the inclined surface is reflected so that the length $P_{1} P_{1}{ }^{\prime}\left(\equiv X_{1}\right)$ of the distorted fringe-circle is recorded on the camera image plane. For simplicity, it is assumed that the reflection angles at $P_{1}$ and $P_{1}{ }^{\prime}$ are equal to $2 \theta_{1}$ (Figure 3 ), and that the recording direction is parallel with z-axis. As the inclination angle $\theta$ of the surface changes from $\theta_{1}$ to $\theta_{2}$, the value of $X$ is changed from $X_{1}{ }^{\prime}$ to $X_{2}{ }^{\prime}$ whereas the value of $Y$ remains the same. For small rotating angle of a relatively small test surface, it can be shown that the angular change $\left(\theta_{2}-\theta_{1}\right)$ can be estimated from the measured values $\left(X_{2}{ }^{\prime}-X_{1}\right)$ using the following expression.

$\theta_{2}-\theta_{1} \approx-\frac{K^{\prime}}{2}\left(\frac{X_{2}^{\prime}-X_{1}^{\prime}}{Y}\right)=-\frac{K^{\prime}}{2} \psi$

where $K^{\prime} \equiv \frac{Y}{D_{c} \xi}$ denotes the optical sensitivity, with $\xi$ defined as $\xi \equiv\left(\frac{d_{c}{ }^{2}-\Delta^{2}}{\Delta^{2}}\right)$.

From Equation (3) and (4), it is readily seen that the rotating angle about the $y$-axis of diffuse and reflective surfaces may be obtained from the amount of distortion of the fringe-circle $\left(X_{2}-X_{I}\right)$ or $\left(X_{2}^{\prime}-X_{I}^{\prime}\right)$ that is measured off the imageplane of the CCD camera; the optical sensitivity factor $K$ or $K^{\prime}$ may be obtained by calibration. 


\section{EXPERIMENTAL ILLUSTRATIONS}

Using the set-up shown in Figure 1, a small (size of $10 \mathrm{~mm}$ by $10 \mathrm{~mm}$ ) object with diffuse surface is mounted on a rotating stage. Figure 4 shows a typical circular grating that is recorded directly off the surface using a CCD camera - the diameter of the innermost circle is approximately $3.0 \mathrm{~mm}$ and that of the third inner circle is approximately $8 \mathrm{~mm}$. . The distortion $\left(X_{2}-X_{1}\right)$ of the fringe-circles along the $x$-axis, denoted by a dimensionless parameter $\psi\left(\equiv \frac{X_{2}-X_{1}}{Y_{2}}\right)$, is plotted against the angular rotation and is shown in Figure 5 - the linear relationship enables determination of the optical sensitivity factor $K$ (Eq. (3)) to enable subsequent use for measuring the angular rotation.

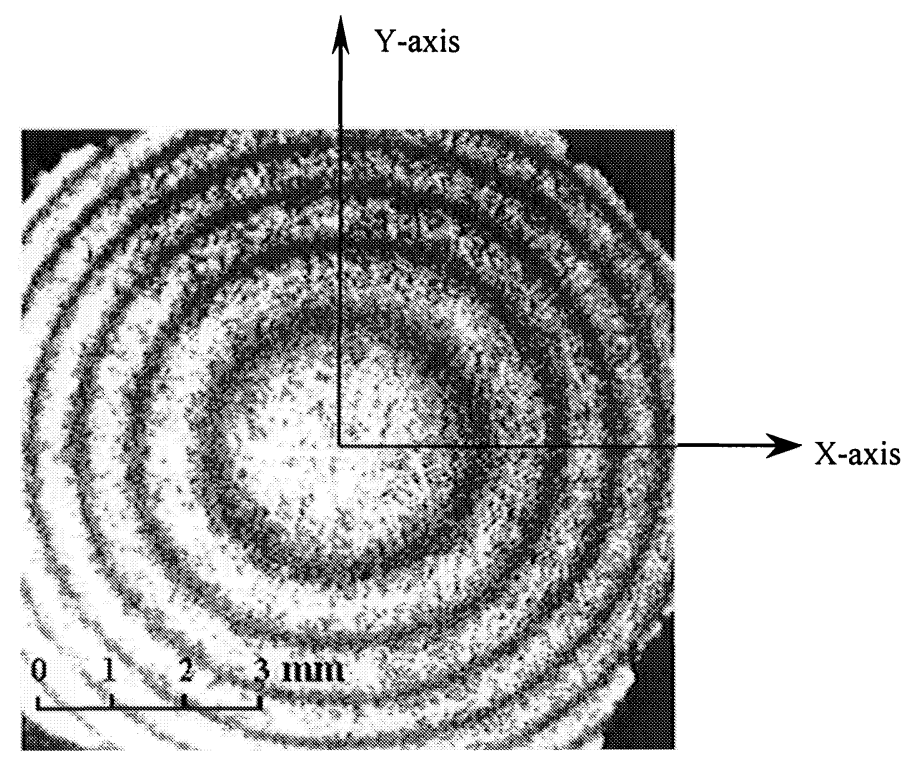

Fig. 4. A typical circular grating observed on the surface

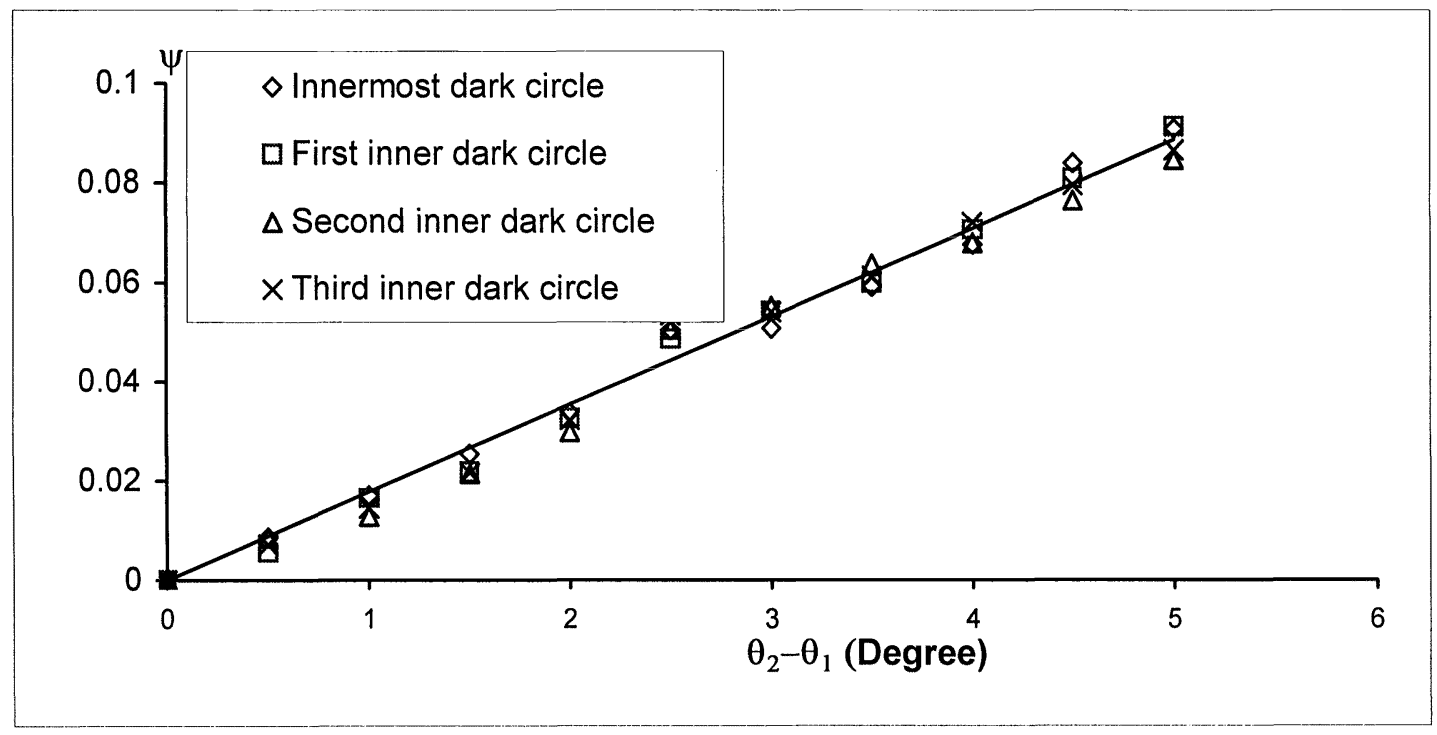

Fig. 5. Linear relationship between $\psi$ and angular rotation of diffuse surface 
For objects a with specularly reflective surface, Figure 6 shows the linear relationship (described by Eq. (4)) between the distortion $\psi\left(\equiv \frac{X_{2}^{\prime}-X_{1}^{\prime}}{Y_{2}^{\prime}}\right)$ of the fringe-circles and angular rotation.

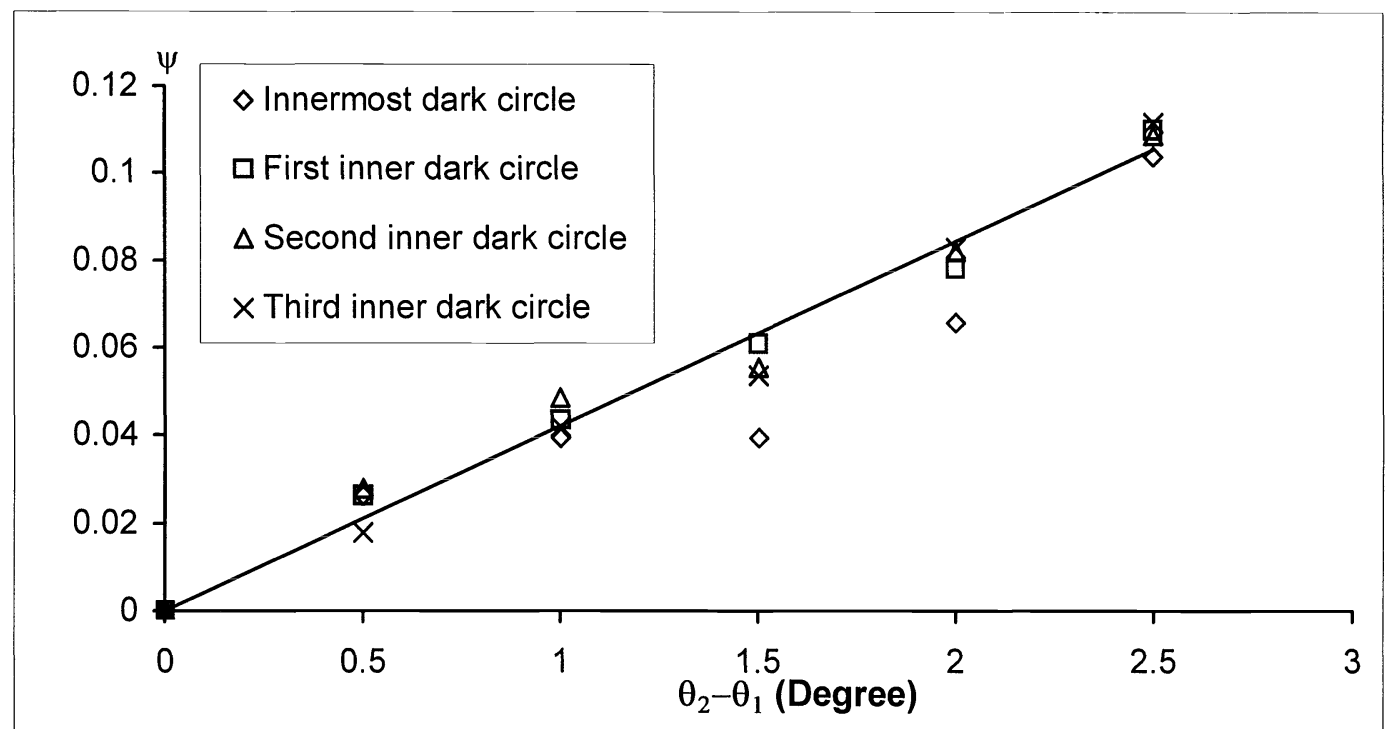

Fig. 6. Linear relationship between $\psi$ and angular rotation of a specularly reflective surface

\section{CONCLUSION}

In this paper, a Michelson-based simple method is described for the generation of a circular optical grating for subsequent projection onto a diffuse or specularly reflective surface. The diameter of the generated optical grating can be easily adjusted using a simple lens placed between the beam-splitting cube and the test surface. The present experimental results showed a linear relationship between the amount of distortion of the fringe-circle and the rotating angle, thus enabling the measurement of small rotating angle of flat surfaces.

\section{REFERENCES}

[1] Collier RJ, Burckhardt CB, Lin LH. Optical holography. New York: Academic Press, 1971.

[2] Erf RK. Holographic nondestructive testing. New York: Academic Press, 1971.

[3] Vest CM. Holographic interferometry. New York: John, Wiley, 1979.

[4] Post D, Han B, Ifju P. High sensitivity Moiré - experimental analysis for mechanics and materials. Berlin: Springer-Verlag, 1994.

[5] Rastogi PK (Ed). Optical measurement techniques and applications. USA: Artech House, 1997.

[6] Rastogi PK, Inaudi D (Ed). Trends in optical nondestructive testing and inspection, UK: Elsevier Press, 2000.

[7] Zhang LY, Qin S, Shang HM. An optical study of the mechanical behavior of the micro-beam of an accelerometer. Proc the Fourth International Conference on Composites Engineering, 6-12 July 1997. Hawaii, USA, p. 877-878.

[8] Huang PS, Kiyono S, Kamada O. Angle measurement based on the internal-reflection effect: a new method. Appl Opt 1992;31(28):6047-6055.

[9] Huang PS, Li Y. Small-angle measurement by use of a single prism. Appl Opt 1998;37( 28):6636-6642.

[10] Tiziani HJ. A study of the use of laser speckle to measure small tilts of optically rough surfaces. Opt Communi. 1972;5(4):271 - 276. 